\title{
Visualization of Multiple FtsZ Rings in Actively Dividing Proplastids of Cultured Bright Yellow-2 Tobacco Cells
}

\author{
Yutaka Miyazawa*, Toshiyuki Mori, Tamaki Kobayashi, Yu Momoyama, \\ Haruko Kuroiwa and Tsuneyoshi Kuroiwa \\ Department of Biological Sciences, Graduate School of Science, \\ University of Tokyo, Hongo, tokyo 113 - 0033, Japan
}

Accepted October 31, 2001

\begin{abstract}
Summary The transfer of stationary-phase cultured tobacco (Nicotiana tabacum L.) Bright Yellow-2 (BY-2) cells into new medium induces active proplastid division. To examine the relationship between $f t s Z$ gene expression and proplastid division, the transcript and protein levels of the fts $Z$ gene in stationary-phase cells and in 12-h-old cells after medium renewal were compared. RNA-gel blot analysis revealed that transcript levels for both types of fts $Z$ genes, fts $Z 1$ and ftsZ2, increased markedly following medium renewal. Similarly, immunoblot analysis using antibodies to lily FtsZ protein showed a remarkable increase in FtsZ protein following medium renewal. Furthermore, immuno-fluorescence microscopic observation revealed that FtsZ proteins appeared as multiple rings transversely aligned in elongated proplastids, which will be fragmented by multiple divisions during culture. These results suggest that FtsZ proteins may have a role in multiple division of proplastids.
\end{abstract}

Key words Cultured tobacco (Nicotiana tabacum) BY-2 cells, FtsZ, Proplastid division.

Plastids are major organelles found in autotrophic plant and algal cells, which are responsible for photosynthesis and for the storage of starch and other molecules. They contain their own genetic material, replicate semiautonomously, and multiply by division. Proplastids are colorless, undifferentiated plastids, which occur in the meristematic regions of the plant, and are considered to be the precursors of other types of plastids, including chloroplasts, etioplasts, and amyloplasts. There are approximately 20 proplastids in each meristematic cell, which proliferate actively to keep pace with cell division. The mechanism of proplastid division has not yet been fully described.

An electron-dense plastid-dividing ring (PD ring) was first identified in the red alga, Cyanidium caldarium RK-1, (Mita et al. 1986); PD rings have been observed subsequently in red, brown, and green algae; mosses; ferns; and higher plants, including tobacco BY-2 cells (Kuroiwa et al. 1998, references therein). PD rings consist of three ring structures-one on the cytosolic surface of outer membrane, one on the stromal surface of inner membrane, and one in the inner-membrane space (Miyagishima et al. 1998). The endosymbiont theory, which proposes that chloroplasts evolved from cyanobacteria, maintains that it is possible for plastids to divide by a system analogous to bacterial cell division. Indeed, homologues of the $f t s Z$ gene, which form a ring structure (FtsZ ring) at the septum during bacterial cell division, have been identified in the nuclear genomes of many plant species and shown to be imported into plastids. Inhibition of $f t s Z$ gene expression resulted in an inhibition of chloroplast division in Arabidopsis and Physcomitrella (Osteryoung et al. 1998, Strepp et al. 1998), indicating that FtsZ proteins may have some role in chloroplast division. However, an involvement of FtsZ protein in proplastid division has not been shown.

The localization of FtsZ proteins in proplastids using fluorescent visualization techniques could provide solid evidence for a relationship between proplastid division and FtsZ protein. As the

* Corresponding author, e-mail: yutakam@postman.riken.go.jp, Present address: RIKEN (The Institute of Physical and Chemical Research), 2-1 Hirosawa, Wako, Saitama 351-0198, Japan 
complexity of highly organized meristematic tissues makes it difficult to analyze the proplastid division, cultured tobacco BY-2 cells were used to analyze proplastid division, which can be induced by renewal of the culture medium (Yasuda et al. 1988). In the current study, the renewal of culture medium was shown to induce $f t s Z$ gene expression and the appearance of multiple Fts $Z$ rings in an elongated proplastid at the early phase of plastid division in BY-2 cells.

Materials and methods

\section{Cell culture and RNA isolation}

The tobacco cell line BY-2, derived from Nicotiana tabacum L. cv. Bright Yellow-2, was propagated on a gyratory shaker at $130 \mathrm{rpm}$ at $26^{\circ} \mathrm{C}$ in the dark. Samples of $5 \mathrm{ml}$ of stationary phase cells were transferred at weekly intervals to $95 \mathrm{ml}$ of a modified Linsmaier and Skoog medium as previously described (Nagata and Kumagai 1999). Total RNA was extracted as previously described (Miyazawa et al. 1999).

\section{Cloning of tobacco ftsZ cDNAs and RNA gel-blot analysis}

Tobacco cDNAs for $f t s Z$ were amplified by PCR using the following technique. Three upstream and 2 downstream degenerated oligonucleotide primers, 5'-GG(inosine)GG(inosine)GG(inosine)GG(inosine)AA(C/T)AA(C/T)GC-3', 5'-AC(inosine)GC(inosine)GC(inosine)ATGGG(A/C/G/T)GG(A/C/G/T)GG-3', 5'-GG(inosine)GA(A/G)CA(A/G)GC(inosine)GC(inosine)GA(A/G)GA3', 5'-CAT(inosine)GC(inosine)GT(inosine)CC(inosine $)(\mathrm{C} / \mathrm{T})(\mathrm{C} / \mathrm{T})(\mathrm{A} / \mathrm{G}) \mathrm{TC}(\mathrm{C} / \mathrm{T}) \mathrm{TTCAT}-3^{\prime}, \quad 5^{\prime}-$ GT(A/G/T)AT(A/G)TTCCA(inosine)AC(A/G/T)AT(inosine)CC-3' were based on conserved amino acid regions of FtsZ proteins of various plants. First strand cDNA and PCR amplification was accomplished using a ReverTra-Dash kit (Toyobo, Osaka, Japan) using $1 \mu \mathrm{g}$ of total RNA isolated from 12-h-old cells as the template. For PCR amplification, the DNA was denatured at $96^{\circ} \mathrm{C}$ for $2 \mathrm{~min}$ in the first cycle, and then at $94^{\circ} \mathrm{C}$ for $1 \mathrm{~min}$ in subsequent cycles. Primer annealing and extension reactions were carried out at $49^{\circ} \mathrm{C}$ or $72^{\circ} \mathrm{C}$ for 1 min each, respectively. After 40 cycles, PCR products were subcloned into pT7-Blue (Novagen, Madison, WI) according to the manufacturer's instructions. The PCR fragment was sequenced, and the database was searched for similar nucleotide sequences using the BLAST algorithm. The amino acid sequences of the cloned fragments for $f t s Z$ had significant identity with known $f t s Z$, including $f t s Z 1$ and $f t s Z 2$, so these fragments were used as probes in further analyses. RNA gel-blot was performed as previously described (Miyazawa et al. 1999).

\section{Immunoblot analysis, and immuno-fluorescence microscopic observations}

Frozen BY-2 cells were homogenized in a buffer of $62.5 \mathrm{mM}$ Tris- $\mathrm{HCl}(\mathrm{pH} 6.8), 10 \%(\mathrm{v} / \mathrm{v})$ glycerol, 2\% (w/v) SDS, and 5\% (v/v) 2-mercaptoethanol, and boiled for $3 \mathrm{~min}$. Immunoblot analysis was performed according to the methods of Mori et al. (2001b). For microscopic observations, collected BY-2 cells were treated in a solution of $1 \%$ Cellulase YC, $0.1 \%$ Pectolyase Y23, and $0.4 \mathrm{M}$ mannitol, pH 5.5 as previously described (Miyazawa et al. 1999) to make protoplasts. These protoplasts were fixed in $3 \%(\mathrm{w} / \mathrm{v})$ paraformaldehyde solution. Immunofluorescent staining was performed according to the methods of Kuroiwa et al. (2001); samples were observed using a fluorescent microscope equipped with phase contrast optics (model BHS-RFK, Olympus, Tokyo).

\section{Results and discussion}

The effects of medium renewal on fts $Z$ gene expression

Earlier work showed that the transfer of BY-2 cells at stationary-phase into new culture medium induced active proplastid division (Yasuda et al. 1988). These changes involve an elongation of 


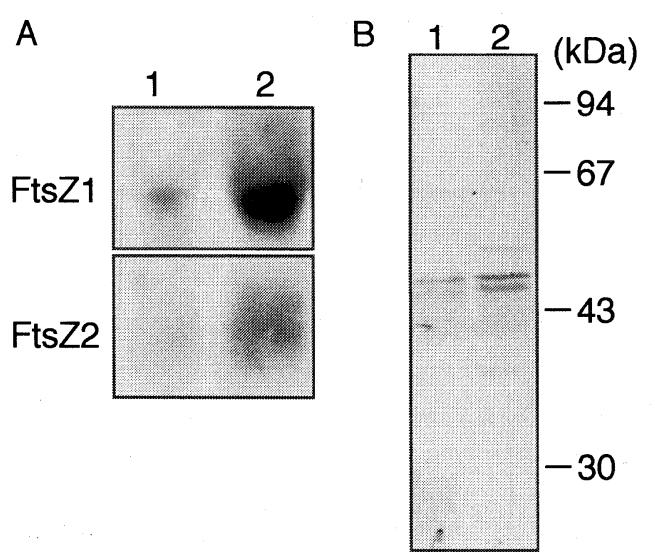

Fig. 1. Effects of medium renewal on $f t s Z$ gene expression. A) RNA-gel blot analysis. Total RNA was extracted from stationary-phase cells and 12-h-old cells and subjected to RNA gel-blot analyses using cDNA fragments of $f t s Z 1$ and $f t s Z 2$ as probes. For detection, each lane was loaded with $10 \mu \mathrm{g}$ of total RNA. lane 1 ; stationary-phase cells, lane 2; 12-h-old cells. B) Immunoblot analysis. $20 \mu \mathrm{g}$ of BY-2 protein were loaded per lane for SDSPAGE. Lanes 1 and 2 correspond to stationary-phase cells and 12-h-old cells, respectively. The arrowhead indicates the protein recognized by antibodies.

plastids, which occurs $12-24 \mathrm{~h}$ post-transfer (Suzuki et al. 1992, Miyazawa et al. 2001). To investigate the relationship between proplastid division and $f t s Z$ gene expression, fts $Z$ genes from cultured BY-2 cells were cloned to determine whether transferring into new medium enhanced the expression of fts $Z$ genes. Transcript levels for both types of fts $Z$, fts $Z 1$ and ftsZ2, were low in stationary-phase cells (Fig. 1A). When these cells were transferred into new medium, accumulation of transcripts for both $f t s Z 1$ and $f t s Z 2$ increased within $12 \mathrm{~h}$. The protein levels for FtsZ2 were examined using antibodies produced against lily FtsZ (LlFtsZ, Mori et al. 2001a,b). The antibodies detected polypeptides from cultured cell extracts, at a molecular mass similar to that of LlFtsZ protein (Fig. 1B). Similarly, protein levels for FtsZ2 increased when cells were transferred into new medium. In red alga Cyanidium caldarium, fts $Z$ gene expression increases just before plastid division (Takahara et al. 2000), and in Lilium longiflorum, FtsZ proteins accumulate at higher levels in young leaves, where active chloroplast division occurs, than in mature leaves (Mori et al. 2001b). As proplastids of BY-2 cells began to elongate prior to division $12 \mathrm{~h}$ after transfer, it is likely that the increased $f t s Z$ gene expression contributed to proplastid division.

\section{Multiple Fts $Z$ rings appear in elongated proplastids}

Previous reports showed that FtsZ proteins were localized to form rings in Arabidopsis, lily, pea, Pelargonium, and tobacco chloroplasts (Kuroiwa et al. 2001, Mori et al. 2001b, Vitha et al. 
2001). Whether FtsZ proteins form ring structures in other actively dividing plastids, such as proplastids, has not yet been investigated, but PD rings have been discovered in other types of plastids in various species (reviewed in Kuroiwa et al. 1998). If the mechanism of plastid division is universal, an FtsZ ring should be observed in other types of dividing plastids. To investigate this hypothesis, the localization of FtsZ protein in proplastids of cultured tobacco BY-2 cells was observed. In BY-2 cells at stationary-phase, where plastids appear as ellipsoidal or spherical in shape, cells contain only one FtsZ ring in each plastid (Fig. 2A). As plastids do not undergo divisions in stationaryphase cells (Yasuda et al. 1988), 2 possibilities for occurrence of FtsZ ring might be considered. The first possibility is that FtsZ ring has some role other than plastid division in stationary-phase cells. The other is that plastid division is arrested at the phase of formation of FtsZ ring. In BY-2 cells, PD rings have not been observed in stationary-phase cells, so far, it is likely that the plastid division is arrested at just before PD ring formation. Also, Kuroiwa et al. (2001) reported that FtsZ rings formed before PD rings appeared. However, the exact role of FtsZ protein in non-dividing plastids remains to be determined. When BY-2 cells were transferred into new medium, proplastids elongate before their multiple divisions, and FtsZ proteins appear as several rings aligned transversely to the direction of elongation (Fig. 2B). Also, weak signals, which may be derived from FtsZ monomers, were detected at other regions. This increase in FtsZ rings is consistent with immunoblot analysis. This arrangement of rings suggests that multiple plastid division planes may occur simultaneously in one elongated proplastid, an observation that is consistent with other studies, which showed that multiple PD rings could be seen using electron microscopy (Kuroiwa 1989). Such multiple rings cannot be observed in stationary-phase cells. Elongated proplastids go on to divide into many pieces $48 \mathrm{~h}$ post-transfer, suggesting that the rings have some role in the determination of division sites. Pleomorphic chloroplasts in the embryonic cap cells of Pelargonium zonale have also been shown to contain multiple FtsZ and PD rings (Kuroiwa et al. 2001). The results of the current study show that FtsZ proteins form multiple ring structures in pre-division proplastids, and that both binary fission and multiple fission of plastids may occur depending on their developmental states.

\section{Acknowledgement}

This work was supported by grants from the Ministry of Education, Culture, Sports, Science, and Technology, of Japan (No. 12440222), and from the Program for Promotion of Basic Research Activities for Innovative Biosciences (PROBRAIN) to T. K.

\section{References}

Kuroiwa, H., Mori, T., Takahara, M., Miyagishima, S. and Kuroiwa, T. 2001. Multiple FtsZ rings in pleomorphic chloroplasts in embryonic cap cells of Pelargonium zonale. Cytologia 66: 227-233.

Kuroiwa, T. 1989. The nuclei of cellular organelles and the formation of daughter organelles by the "plastid-dividing ring." Bot. Mag. Tokyo 102: 291-329.

-, Kuroiwa, H., Sakai, A., Takahashi, H., Toda, K. and Itoh, R. 1998. The division apparatus of plastids and mitochondria. Int. Rev. Cytol. 181: 1-41

-, Takahara, M., Miyagishima, S., Ohashi, Y., Kawamura, F. and Kuroiwa, H. 1999. The FtsZ protein is not localized on outer plastid dividing rings. Cytologia 64: 333-342.

Mita, T., Kanbe, T., Tanaka, K. and Kuroiwa, T. 1986. A ring structure around the dividing plane of the Cyanidium caldarium chloroplast. Protoplasma 130: 211-213.

Miyagishima, S., Itoh, R., Toda, K., Takahashi, H., Kuroiwa, H. and Kuroiwa, T. 1998. Identification of a triple-ring structure involved in plastid division in the primitive red alga Cyanidioshyzon merolae. J. Electron Microsc. 47: 269-272.

Miyazawa, Y., Sakai, A., Miyagishima, S., Takano, H., Kawano, S. and Kuroiwa, T. 1999. Auxin and cytokinin have opposite effects on amyloplast development and the expression of starch synthesis genes in cultured tobacco BY-2 cells. 
Plant Physiol. 121: 461-469.

-, -, Kawano, S. and Kuroiwa, T. 2001. Differential regulation of starch synthesis gene expression during amyloplast development in cultured tobacco BY-2 cells. J. Plant Physiol. 158: 1077-1084.

Mori, T., Takahara, M., Miyagishima, S., Kuroiwa, H. and Kuroiwa, T. 2001a. Visualization of FtsZ rings in plastids of the microspore in Lilium longiflorum. Cytologia 66: 113-115.

—, Kuroiwa, H., Takahara, M., Miyagishima, S. and Kuroiwa, T. 2001b. Visualization of an FtsZ ring in chloroplasts of Lilium longiflorum leaves. Plant Cell Physiol. 42: 555-559.

Nagata, T. and Kumagai, F. 1999. Plant cell biology through the window of the highly synchronized tobacco BY-2 cell line. Methods Cell Sci. 21: 123-127.

Osteryoung, K. W., Stokes, K. D., Rutherford, S. M., Percival, A. L. and Lec, W. Y. 1998. Chloroplast division in higher plants requires members of two funcitonally divergent gene families with homology to bacterial ftsZ. Plant Cell 10: 1991-2004.

Strepp, R., Scholz, S., Kruse, S., Speth, V. and Reski, R. 1998. Plant nuclear gene knockout reveals a role in plastid division for the homolog of the bacterial cell division protein FtsZ, an ancestral tublin. Proc. Natl. Acad. Sci. USA 95: $4368-4373$.

Suzuki, T., Kawano, S., Sakai, A., Fujie, M., Kuroiwa, H., Nakamura, H. and Kuroiwa, T. 1992. Preferential mitochondrial and plastid DNA synthesis before multiple cell divisions in Nicotiana tabacum. J. Cell Sci. 103: 831-837.

Takahara, M., Takahashi, H., Matsunaga, S., Sakai, A., Kawano, S. and Kuroiwa, T. 2000. Isolation, characterization, and chromosomal mapping of an $f t s Z$ gene from the unicellular primitive red alga Cyanidium caldarium RK-1. Curr. Genet. 37: 143-151.

Vitha, S., McAndrew, R. S. and Osteryoung, K. W. 2001. FtsZ ring formation at the chloroplast division sites in plants. J. Cell Biol. 153: 111-119.

Yasuda, T., Kuroiwa, T. and Nagata, T. 1988. Preferential synthesis of plastid DNA and increased replication of plastids in cultured tobacco cells following medium renewal. Planta 174: 235-241. 